УДК 621.315 .592

\title{
Влияние примеси никеля на гальваномагнитные свойства и электронную структуру РbTe
}

\author{
( Е.П. Скипетров ${ }^{1,2}$, Б.Б. Ковалев ${ }^{1}$, И.В. Шевченко ${ }^{1}$, А.В. Кнотько ${ }^{2,3}$, В.Е. Слынько ${ }^{4}$ \\ ${ }^{1}$ Московский государственный университет им. М.В. Ломоносова (физический фракультет), \\ 119991 Москва, Россия \\ ${ }^{2}$ Московский государственный университет им. М.В. Ломоносова (факультет наук о материалах), \\ 119991 Москва, Россия \\ ${ }^{3}$ Московский государственный университет им. М.В. Ломоносова (химический фракультет), \\ 119991 Москва, Россия \\ ${ }^{4}$ Институт проблем материаловедения им. И.Н. Францевича Национальной академии наук Украины, \\ 58001 Черновцы, Украина \\ E-mail: skip@mig.phys.msu.ru
}

Поступила в Редакцию 15 апреля 2020 г.

В окончательной редакции 20 апреля 2020 г.

Принята к публикации 27 апреля 2020 г.

Исследованы фазовый и элементный состав и гальваномагнитные свойства (в диапазоне температур $4.2 \leq T \leq 300 \mathrm{~K}$, в магнитных полях $B \leq 7$ Тл) сплавов $\mathrm{Pb}_{1-y} \mathrm{Ni}_{y}$ Те при вариации концентрации примеси никеля вдоль монокристаллического слитка, синтезированного методом Бриджмена-Стокбаргера. Показано, что растворимость никеля не превышает 0.35 мол\%. Обнаружены аномальные температурные зависимости коэффициента Холла и температурные и полевые зависимости магнитосопротивления. Для объяснения результатов сделано предположение о возникновении инверсионного слоя $n$-типа проводимости на поверхности образцов и существовании нескольких конкурирующих механизмов проводимости в образцах. Предложена модель электронной структуры $\mathrm{Pb}_{1-y} \mathrm{Ni}_{y} \mathrm{Te}$, предполагающая пиннинг уровня Ферми в пределах примесной зоны $\mathrm{Ni}$, расположенной на краю валентной зоны и смещающейся в глубь нее с ростом температуры.

Ключевые слова: сплавы $\mathrm{Pb}_{1-y} \mathrm{Ni}_{y} \mathrm{Te}$, гальваномагнитные эффекты, электронная структура, примесная зона никеля.

DOI: $10.21883 /$ FTP.2020.10.49932.9417

\section{1. Введение}

Легирование сплавов на основе теллурида свинца примесями переходных $3 d$-металлов приводит к появлению в основном резонансных донорных примесных уровней в электронном спектре этих материалов [1]. $\mathrm{B}$ РbTe уровни примесей из первой половины ряда $(\mathrm{Sc}, \mathrm{Ti}, \mathrm{V}, \mathrm{Cr})$ находятся в окрестности дна зоны проводимости [2-5]. Первый из уровней $3 d$-примесей с переменной валентностью, расположенный в валентной зоне, - уровень $\mathrm{Fe}$ - в РbТе находится под самым ее потолком [6]. При увеличении содержания олова в сплавах $\mathrm{Pb}_{1-x} \mathrm{Sn}_{x} \mathrm{Te}$ он движется в глубь „легкой“ валентной зоны с экстремумами в точках $L$ зоны Бриллюэна и может достичь потолка „тяжелой“ валентной $\Sigma$-зоны при некотором зависящем от температуры критическом содержании олова $(x=0.60-0.65$ при температуре $T=4.2 \mathrm{~K}$ ) [7,8]. Это обстоятельство представляет интерес с точки зрения возможности повышения термоэлектрической эффективности материалов на основе полупроводников $\mathrm{A}^{\mathrm{IV}} \mathrm{B}^{\mathrm{VI}}$ методами, „зоной инженерии“ [9-19]. В частности, в этом случае можно надеяться на достижение максимального синергетического эффекта в $\mathrm{Pb}_{1-x-y} \mathrm{Sn}_{x} \mathrm{Fe}_{y} \mathrm{Te}$ в результате объединения преимуществ двух ее основных направлений: пересечения экстремумов „легкой“ и „тяжелой“ валент- ных зон в электронном спектре [9-15] и присутствия резонансного уровня вблизи уровня Ферми [13-19].

Подобными свойствами могут обладать и сплавы, легированные другими примесями из второй половины ряда переходных $3 d$-металлов. Сопоставление характера изменения энергетического положения известных в настоящее время уровней $\mathrm{Sc}, \mathrm{Ti}, \mathrm{V}, \mathrm{Cr}, \mathrm{Mn}, \mathrm{Fe}$ в $\mathrm{PbTe}$ и в хорошо изученных соединениях $\mathrm{A}^{\mathrm{III}} \mathrm{B}^{\mathrm{V}}, \mathrm{A}^{\mathrm{II}} \mathrm{B}^{\mathrm{VI}}[20]$ позволяет предполагать, что уровни Со и $\mathrm{Ni}$ в $\mathrm{PbTe}$ так же, как и уровень $\mathrm{Fe}$, могут находиться в окрестности потолка валентной зоны. Однако систематические исследования сплавов на основе РbТе, легированных Со и $\mathrm{Ni}$, до сих пор не проводились, а известные нам экспериментальные данные по гальваномагнитным свойствам и зонной структуре этих полупроводников крайне немногочисленны [21-27].

Поскольку нелегированные кристаллы РbТе имеют $p$-тип проводимости, а почти во всех исследованных случаях кристаллы $\mathrm{Pb}_{1-y} \mathrm{Co}_{y} \mathrm{Te}$ и $\mathrm{Pb}_{1-y} \mathrm{Ni}_{y} \mathrm{Te}$ характеризовались проводимостью $n$-типа, считалось, что примесные центры имеют донорный характер. Предполагалось, что они представляют собой электрически активные ионы $3^{+}$, расположенные в междоузлиях (в тетраэдрических пустотах гранецентрированной кубической решетки РbTe) [21-23]. По данным электрофизических и оптических исследований $[21,22]$, глубокий уровень Со 


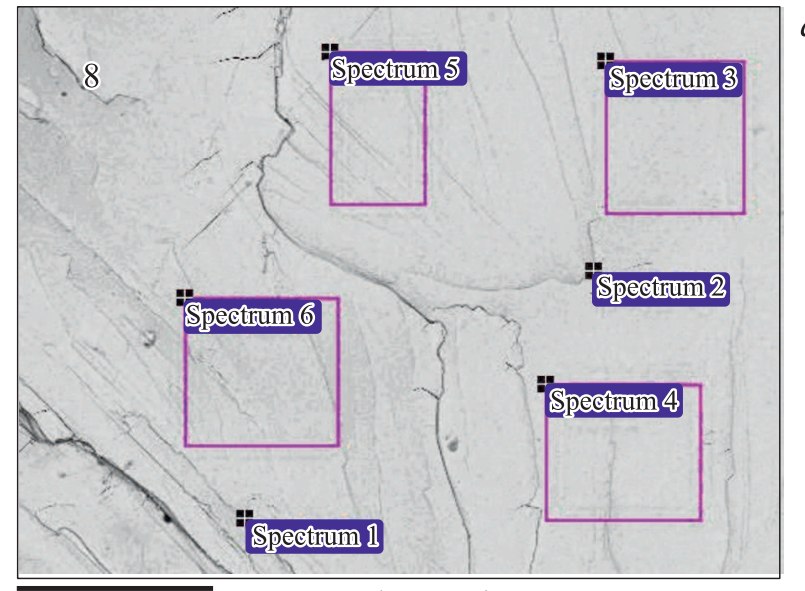

$100 \mu \mathrm{m}$

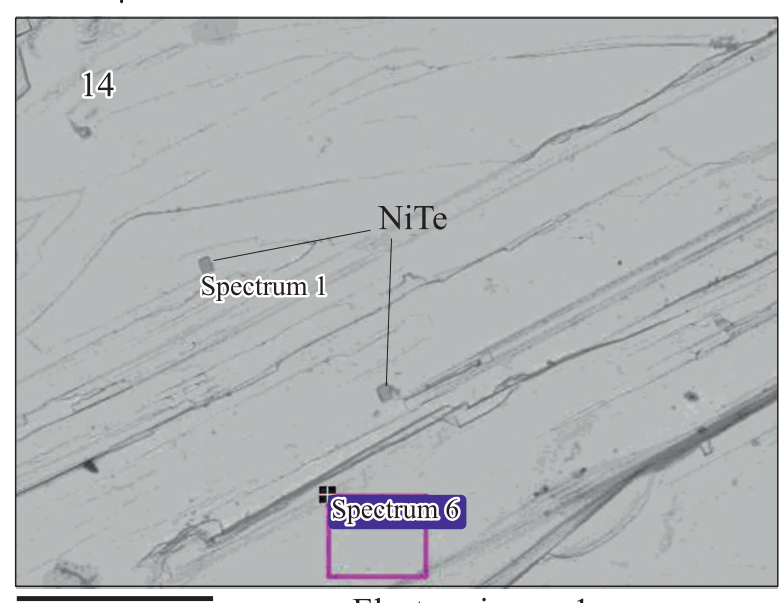

$100 \mu \mathrm{m}$

Electron image 1 $a$
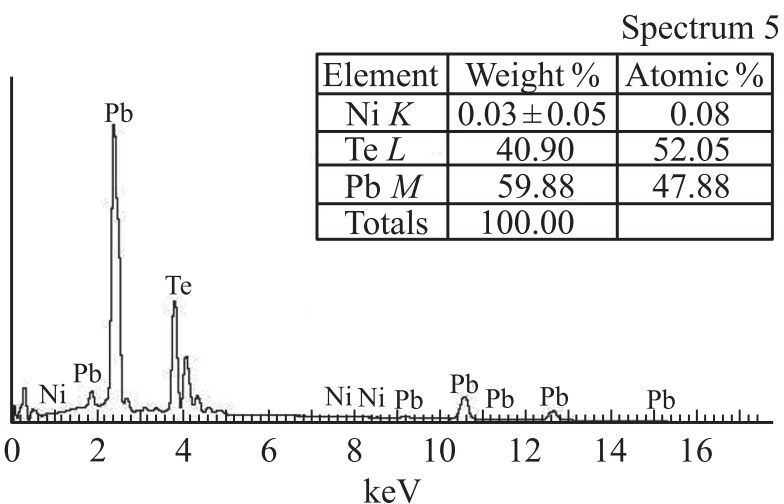

Full Scale 51944 cts Cursor:-0.001 (2033 cts)

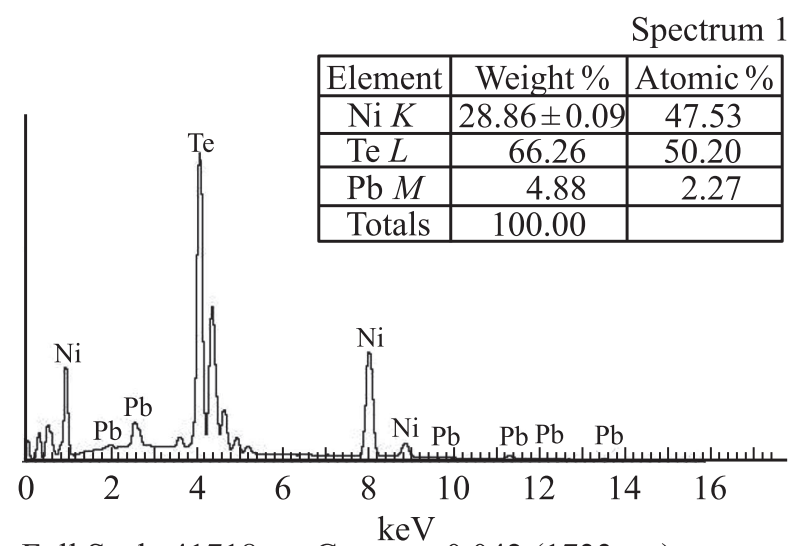

Full Scale 41718 cts Cursor:-0.042 (1733 cts)

Рис. 1. Фотографии поверхностей сколов и рентгеноэмиссионные спектры для образцов 8 и 14 , полученные с помощью растрового электронного микроскопа.

при гелиевых температурах должен находиться в запрещенной зоне, на 60 мэВ ниже дна зоны проводимости, а резонансный уровень $\mathrm{Ni}$ - в зоне проводимости и смещаться вверх по энергии при увеличении температуры.

В настоящей работе исследованы гальваномагнитные эффекты (диапазон температур $4.2 \leq T \leq 300 \mathrm{~K}$, интервал магнитных полей $B \leq 7$ Тл) в образцах из монокристаллического слитка $\mathrm{Pb}_{1-y} \mathrm{Ni}_{y} \mathrm{Te}(y=0.01)$ при вариации концентрации никеля вдоль слитка. Основными целями были обнаружение качественных эффектов, указывающих на присутствие примесного уровня никеля в электронном спектре и определение его энергетического положения в сплавах.

\section{2. Образцы, методика эксперимента}

В работе исследовались образцы из монокристаллического слитка $\mathrm{Pb}_{1-y} \mathrm{Ni}_{y} \mathrm{Te}$ с номинальной концентрацией никеля $y=0.01$, синтезированного вертикальным методом Бриджмена-Стокбаргера из смеси предварительно синтезированных $\mathrm{PbTe}$ и $\mathrm{NiTe}(\mathrm{Pb}$ - 99.9999\%, Te -
99.9999\%, $\mathrm{Ni}$ - 99.6\%). Методика подготовки исходных компонентов и синтеза монокристаллов подробно описана в работе [28]. С помощью струнной резки слитки разрезались перпендикулярно оси роста, совпадающей с точностью до нескольких угловых градусов с кристаллографическим направлением $\langle 111\rangle$, на 20 шайб толщиной $\sim 1.5$ мм. Номера шайб используются далее как номера исследованных образцов.

Фазовый состав, однородность образцов и распределение никеля вдоль слитка исследовались методом рентгенофлюоресцентного микроанализа на сканирующем электронном микроскопе LEO SUPRA 50VP (LEO Carl Zeiss SMT Ltd, Германия) с системой микроанализа INCA Energy+ (Oxford Instruments, Англия). В ряде образцов обнаружены микроскопические включения второй фазы, близкой по составу к соединению NiTe, но основная монокристаллическая фаза, занимающая почти весь объем образцов, сохраняла высокую однородность (рис. 1).

Распределение никеля по длине слитка прогнозировалось с помощью эмпирической формулы, предложенной в работах $[29,30]$ на основе многочисленных данных 


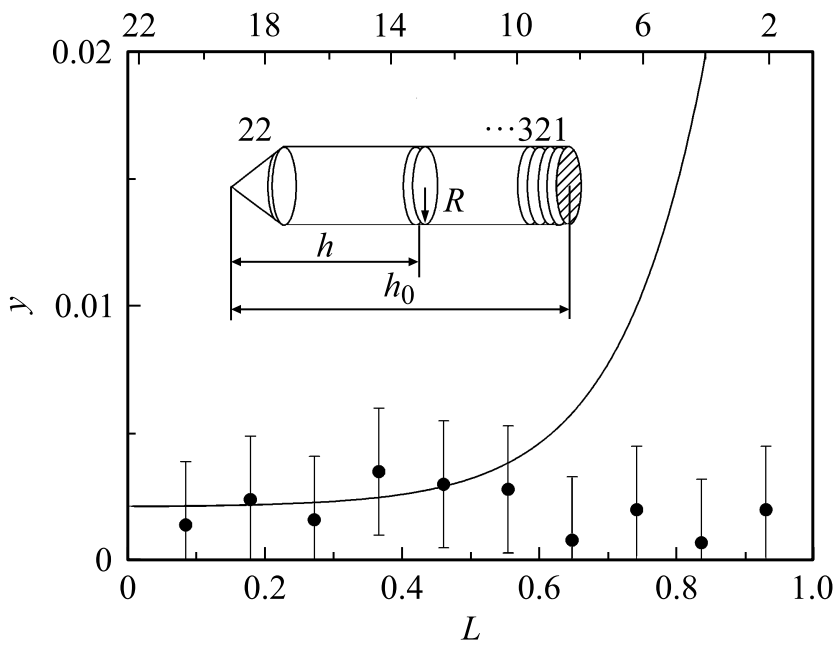

Рис. 2. Распределение примеси никеля по длине монокристаллического слитка $\mathrm{Pb}_{1-y} \mathrm{Ni}_{y} \mathrm{Te}(y=0.01)$ : точки - данные рентгенофлюоресцентного микроанализа, линия - прогноз распределения, построенный по формуле (1).

рентгенофлюоресцентного микроанализа по распределению примесей в полупроводниках группы $\mathrm{A}^{\mathrm{IV}} \mathrm{B}^{\mathrm{VI}}$ (рис. 2):

$$
y(L)=y_{0}+A_{1} \exp \left[\left(L-L_{0}\right) / t_{1}\right]+A_{2} \exp \left[\left(L-L_{0}\right) / t_{2}\right],
$$

где $L=h / h_{0}$ - относительная координата шайбы, $h-$ расстояние от начала слитка до шайбы, $h_{0}$ - полная длина слитка, $y_{0}, A_{1}, A_{2}, L_{0}, t_{1}, t_{2}$ - безразмерные подгоночные параметры.

Ранее подобные расчетные данные с хорошей точностью совпадали с данными рентгенофлюоресцентного анализа по распределению олова в сплавах $\mathrm{Pb}_{1-x-y} \mathrm{Sn}_{x} \mathrm{Cr}_{y} \mathrm{Te}$ и $\mathrm{Pb}_{1-x-y} \mathrm{Sn}_{x} \mathrm{~V}_{y} \mathrm{Te}$, в которых величина $x$ монотонно увеличивалась при движении вдоль слитков $[1,31,32]$. Однако для распределения примесей $\mathrm{Cr}$ и V согласие экспериментальных данных с прогнозом по формуле (1) наблюдалось только в начале и середине слитков, а в конце слитков концентрация примеси выходила на насыщение при 1-1.5 мол\%, что указывало на достижение предела растворимости примеси. В случае $\mathrm{Pb}_{1-y} \mathrm{Ni}_{y} \mathrm{Te}$ ситуация оказалась еще хуже: согласие с прогнозом наблюдается только в начале слитка, где концентрация $\mathrm{Ni}$, возможно, несколько увеличивается, достигая величины 0.35 мол\%. Затем она, по-видимому, даже уменьшается. При этом ошибка ее экспериментального определения почти во всех образцах превышает саму величину концентрации никеля. Поэтому построить адекватную кривую распределения никеля вдоль слитка не удается.

Для исследования гальваномагнитных эффектов из шайб вырезались образцы в виде прямоугольных параллелепипедов с типичными размерами $4.0 \times 0.7 \times 0.7 \mathrm{Mм}$. Перед монтажом образцы травились в растворе брома в бромистоводородной кислоте и тщательно промы- вались этиловым спиртом и дистиллированной водой. Электрические контакты к образцам изготавливались из платиновой проволоки диаметром 0.03 мм. Токовые контакты припаивались к торцам образцов микропаяльником с использованием припоя In $+4 \% \mathrm{Ag}+1 \% \mathrm{Au}$, a потенциальные контакты приваривались электроискровым методом.

Для каждого образца четырехзондовым методом на постоянном токе исследовались температурные зависимости удельного сопротивления $\rho$, относительного поперечного магнитосопротивления $\Delta \rho / \rho_{0}$ и коэффициента Холла $R_{\mathrm{H}}$ в слабых магнитных полях $(4.2 \leq T \leq 300 \mathrm{~K}$, $B \leq 0.07$ Тл), а также полевые зависимости $\Delta \rho / \rho_{0}(B)$ при $T=4.2 \mathrm{~K}$ в магнитных полях до 7 Тл.

\section{3. Температурные и полевые зависимости гальваномагнитных параметров}

Установлено, что температурные зависимости всех исследованных гальваномагнитных параметров изменяются вдоль слитка регулярным образом и имеют аномальный для сплавов на основе РbТе вид. Так, на температурных зависимостях удельного сопротивления обнаружены два активационных участка - низкотемпературный $(T<25 \mathrm{~K})$ и высокотемпературный $(T>100 \mathrm{~K})$, между которыми сопротивление изменяется „металлическим“ образом (рис. 3,a). При движении вдоль слитка (от образца 20 к образцу 4) удельное сопротивление при гелиевых температурах сначала (в образцах 20-10) уменьшается более чем на 2 порядка, а затем (в образцах 10-4) увеличивается до первоначального значения. При этом амплитуда и протяженность „металлического“ участка сначала увеличиваются, а затем уменьшаются, а амплитуда и протяженность высокотемпературного активационного участка - наоборот. Параметры активационных участков почти не меняются, но в образце 10 низкотемпературный активационный участок пропадает.

Температурные зависимости коэффициента Холла $R_{\mathrm{H}}(T)$ всех образцов имеют качественно одинаковый и совершенно необычный вид (рис. $3, b$ ). В широком диапазоне температур от гелиевой до $\sim 80 \mathrm{~K}$ коэффициент Холла имеет отрицательный знак и почти не меняется. Затем происходит быстрое уменьшение абсолютной величины и $n-p$-инверсия знака $R_{\mathrm{H}}$ в узком интервале температур 170-220 К. После этого величина $R_{\mathrm{H}}$ растет, достигает максимума и даже несколько уменьшается в ряде образцов. Необычность этих зависимостей $R_{\mathrm{H}}(T)$ связана с двумя обстоятельствами. Во-первых, абсолютная величина коэффициента Холла в области низких температур во всех образцах аномально велика $\left(\left|R_{\mathrm{H}}\right|=7 \cdot 10^{2}-10^{4} \mathrm{~cm}^{3} /\right.$ Кл $)$, что в случае электронной зонной проводимости соответствует аномально низким концентрациям свободных электронов $n=7 \cdot 10^{14}-10^{16} \mathrm{~cm}^{-3}$. Во-вторых, инверсия типа проводимости при повышении температуры, но из $p$ - в $n$-тип 

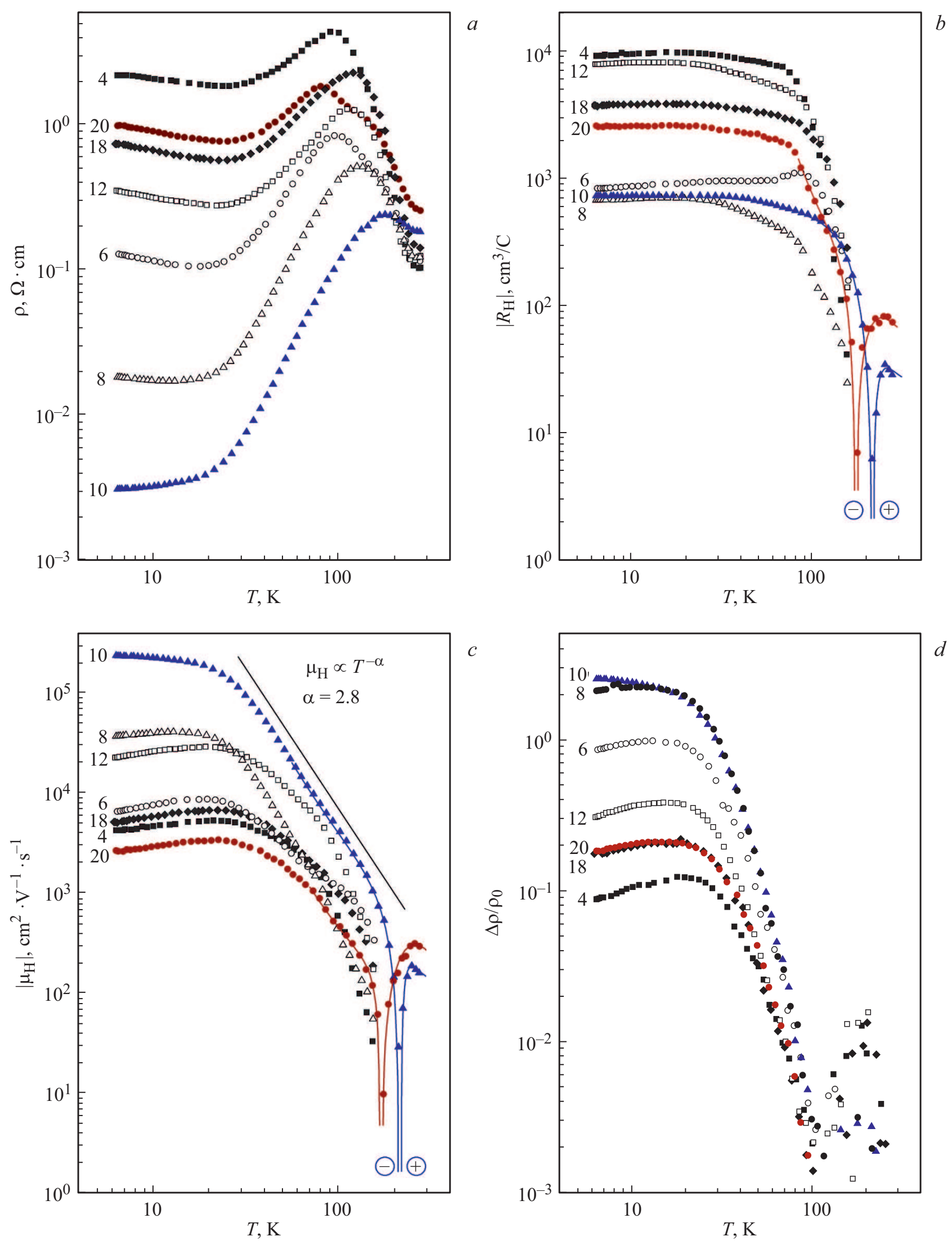

Рис. 3. Температурные зависимости удельного сопротивления $(a)$, коэффициента Холла $(b)$, холловской подвижности $(c)$ и относительного поперечного магнитосопротивления $(d) \mathrm{Pb}_{1-y} \mathrm{Ni}_{y} \mathrm{Te}$ (указаны номера образцов). 

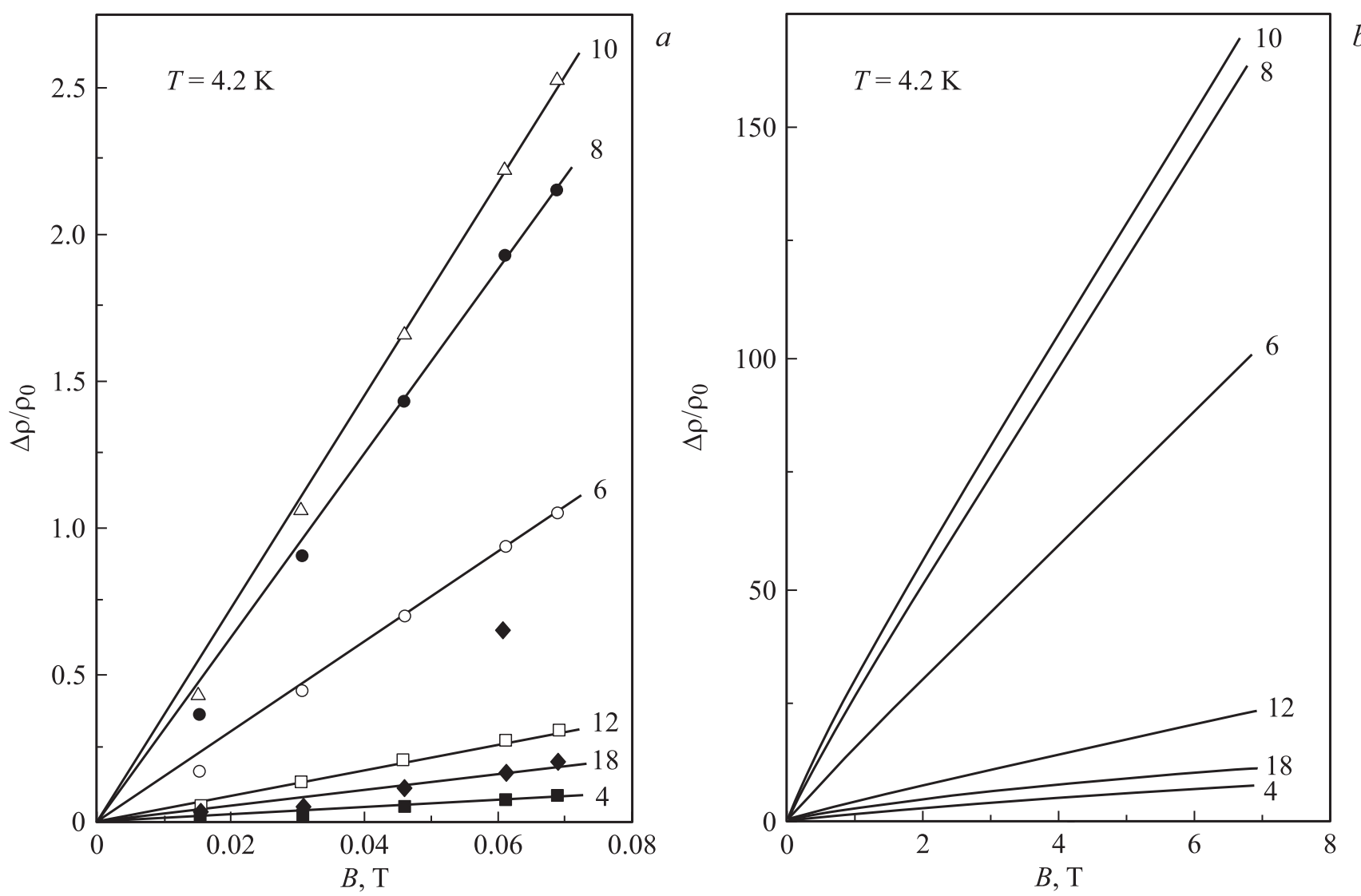

Рис. 4. Полевые зависимости относительного поперечного магнитосопротивления в $\mathrm{Pb}_{1-y} \mathrm{Ni}_{y}$ Tе (указаны номера образцов).

и при несколько более высоких температурах, является вполне обычным явлением в сплавах на основе теллурида свинца и объясняется переходом к собственной проводимости в случаях, когда подвижность электронов заметно превышает подвижность дырок. Однако, насколько нам известно, $n-p$-конверсия в этих материалах ранее не наблюдалась.

Поведение холловской подвижности $\mu_{\mathrm{H}}=R_{\mathrm{H}} / \rho$ при низких температурах противоположно поведению сопротивления - в образцах от 20-го до 10-го она растет на 2 порядка, а в образцах от 10-го до 4-го возвращается обратно (рис. $3, c$ ). При этом зависимости $\mu_{\mathrm{H}}(T)$ качественно повторяют зависимости $R_{\mathrm{H}}(T)$. Однако при $T<25 \mathrm{~K}$ наблюдается уменьшение подвижности при понижении температуры, а ее величины в основном находятся в диапазоне $3 \cdot 10^{3}-4 \cdot 10^{4} \mathrm{~cm}^{2} /(\mathrm{B} \cdot \mathrm{c})$ и выглядят явно ниже значений, типичных для зонной проводимости. Только в самом низкоомном образце 10, в котором пропадает низкотемпературный активационный участок сопротивления, подвижность резко увеличивается, достигая нормального для зонной проводимости значения $\mu_{\mathrm{H}} \approx 2 \cdot 10^{5} \mathrm{~cm}^{2} /(\mathrm{B} \cdot \mathrm{c})$. В области высоких температур показатель степени температурной зависимости подвижности $\left(\mu_{\mathrm{H}} \propto T^{-\alpha}\right)$ изменяется от 1.8 до 2.8 , что характерно для рассеяния на акустических фононах в полупроводниках $\mathrm{A}^{\mathrm{IV}} \mathrm{B}^{\mathrm{VI}}$.
Наконец, в отличие от всех исследованных ранее сплавов на основе $\mathrm{PbTe}$ с примесями переходных металлов, в сплавах $\mathrm{Pb}_{1-y} \mathrm{Ni}_{y} \mathrm{Te}$ обнаружена сильная зависимость сопротивления от магнитного поля. Высокие значения магнитосопротивления практически во всех образцах позволили получить температурные зависимости относительного магнитосопротивления $\Delta \rho / \rho_{0}$ в широком диапазоне температур (рис. $3, d$ ). При гелиевых температурах относительное изменение сопротивления в поперечном магнитном поле $B \approx 0.07$ Тл достигает значения $\Delta \rho / \rho_{0} \approx 2.5$ в отмеченном выше образце 10 (рис. $3, d)$. При движении от этого образца к началу или к концу слитка величина эффекта постепенно уменьшается более, чем на порядок. Температурные зависимости $\Delta \rho / \rho_{0}$ в целом похожи на температурные зависимости холловской подвижности. По мере приближения к точке $n-p$-инверсии относительное магнитосопротивление быстро падает на 3 порядка и в области высоких температур не поддается надежному определению.

Еще одна аномалия обнаружена при исследовании полевых зависимостей относительного поперечного магнитосопротивления при гелиевых температурах: в слабых магнитных полях эти зависимости во всех образцах оказались практически линейными (рис. 4,a). Более того, эта линейность сохраняется и в сильных магнитных полях вплоть до 7 Тл, а величина эффекта достигает ре- 

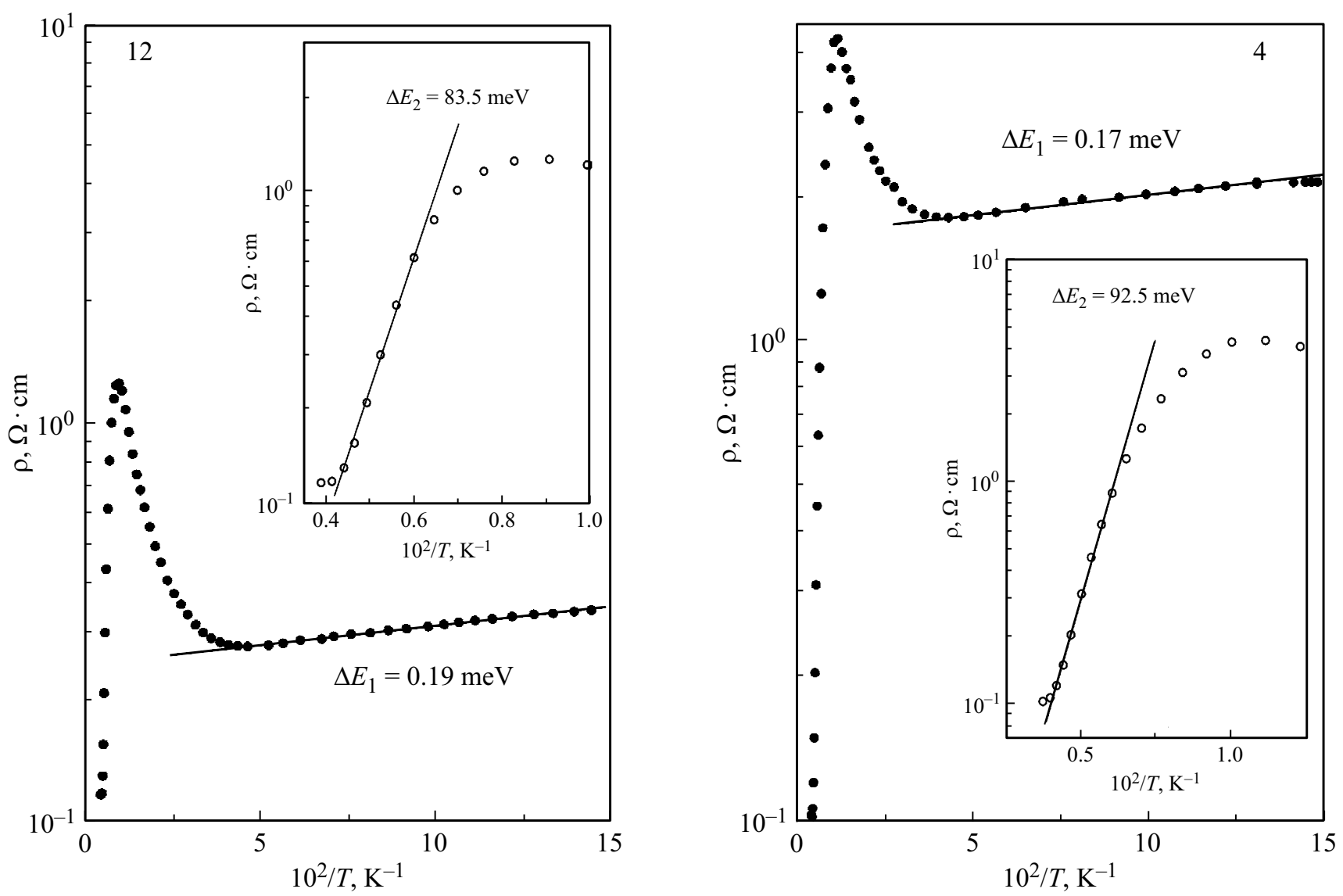

Рис. 5. Температурные зависимости удельного сопротивления образцов 4 и 12 .

кордного значения $\Delta \rho / \rho_{0} \approx 170$ (рис. $4, b$ ). Эти результаты противоречат стандартной теоретической полевой зависимости относительного изменения сопротивления в полупроводнике с одним типом носителей заряда $\left(\Delta \rho / \rho_{0} \propto \mu^{2} B^{2}\right.$ с возможностью насыщения зависимости в сильных магнитных полях). Это обстоятельство, так же как и аномально высокие значения магнитосопротивления, может указывать на присутствие нескольких типов носителей заряда в исследованных образцах.

\section{4. Обсуждение экспериментальных результатов}

\section{1. Характер изменения гальваномагнитных параметров вдоль слитка}

Представленные выше экспериментальные результаты показывают, что в сплавах $\mathrm{Pb}_{1-y} \mathrm{Ni}_{y} \mathrm{Te}$ наблюдается целый ряд аномалий, а их гальваномагнитные свойства принципиально отличаются от свойств нелегированных сплавов на основе РbТе и сплавов, легированных другими примесями переходных $3 d$-металлов. Выделим и обсудим основные полученные результаты.

Последовательное изменение гальваномагнитных параметров при гелиевых температурах и даже темпе- ратуры инверсии знака коэффициента Холла, а также качественно одинаковый вид температурных зависимостей каждого параметра во всех образцах, скорее всего, связаны с характером изменения концентрации никеля и небольшим диапазоном ее изменения вдоль слитка. Среди всех образцов выделяется образец 10 из середины слитка, характеризующийся минимальными величинами сопротивления и модуля коэффициента Холла, максимальными значениями подвижности и магнитосопротивления, а также отсутствием низкотемпературного активационного участка на зависимости $\rho(T)$. Можно предположить, что немонотонный, „возвратный“ характер изменения параметров образцов вдоль слитка обусловлен тем, что концентрация никеля сначала растет, примерно в середине слитка выходит на максимум, достигая предела растворимости, и затем уменьшается.

Температурные зависимости удельного сопротивления исследованных образцов, перестроенные в масштабе $\rho(100 / T)$, были использованы для определения низкотемпературной и высокотемпературной энергий активации $\Delta E_{1}$ и $\Delta E_{2}$ (рис. 5). С этой целью активационные участки аппроксимировались прямыми линиями в соответствии с зависимостями $\rho \propto \exp \left(\Delta E_{1,2} / k T\right)$. Величина $\Delta E_{1}$ во всех образцах, за исключением образца 10 , в котором активационный участок отсутствует, находится 
на уровне $(0.20 \pm 0.05)$ мэВ и почти не меняется вдоль слитка. Такие значения энергии активации вряд ли могут соответствовать примесной ионизации и связаны, скорее всего, с активационной проводимостью по примесной зоне.

Значения высокотемпературной энергии активации также нерегулярно меняются от образца к образцу, $\Delta E_{2}=(85 \pm 20)$ мэВ, и соответствуют величине, которая чуть меньше половины ширины запрещенной зоны $\mathrm{PbTe}$. С учетом низкой точности ее определения по достаточно коротким активационным участкам (см. вставки на рис. 5), она могла бы быть связана с собственной ионизацией носителей заряда. Однако в этом случае должна происходить $p-n$-, а не $n-p$-инверсия знака коэффициента Холла, как в нашем случае. Кроме того, с ростом температуры вслед за проводимостью по примесной зоне должна следовать примесная проводимость, а затем уже собственная. Участок примесной ионизации на зависимостях $\rho(100 / T)$ может отсутствовать только при совпадении энергии глубокого примесного уровня с краями разрешенных зон или с серединой запрещенной зоны.

Необходимо также отметить, что на самой верхней границе исследованного нами температурного диапазона на зависимостях $R_{\mathrm{H}}(T)$, как уже отмечалось, наблюдался максимум (см. рис. $3, b$ ), который может быть связан с переходом к собственной ионизации с возможной последующей $p-n$-инверсией типа проводимости.

Отрицательный знак коэффициента Холла при низких температурах в простейшем случае может быть связан с электронной зонной проводимостью. Тогда в случае одного типа носителей заряда во всех исследованных образцах уровень Ферми должен находиться в зоне проводимости и почти не изменяться до $\sim 80 \mathrm{~K}$. Для проверки этого предположения для всех образцов по величинам коэффициента Холла при гелиевых температурах рассчитаны концентрации электронов в зоне проводимости как $n=1 / e\left|R_{\mathrm{H}}\right|(e-$ элементарный заряд), а затем и положение уровня Ферми относительно дна зоны проводимости в рамках двухзонного закона дисперсии Кейна [33]. Оказалось, что при вариации концентрации электронов в образцах от $7 \cdot 10^{14}$ до $10^{16} \mathrm{~cm}^{-3}$ энергия Ферми относительно дна зоны проводимости вдоль слитка должна немонотонно меняться в диапазоне от 0.2 до 1.2 мэВ. Такие низкие значения этих величин могут наблюдаться только в случае пинннга уровня Ферми резонансным примесным уровнем вблизи дна зоны проводимости, а сохраняться почти до температуры жидкого азота - только при движении резонансного уровня в точности параллельно дну зоны проводимости при увеличении температуры, что не характерно для глубоких примесных уровней. Кроме того, в этом случае трудно объяснить $n-p$-инверсию знака коэффициента Холла и переход к дырочному типу проводимости в широком диапазоне температур, рекордную величину и линейную полевую зависимость магнитосопротивления, а также низкую подвижность электронов при низких температурах.

Альтернативным объяснением отрицательного знака коэффициента Холла при низких температурах и $n$-p-инверсии знака коэффициента Холла при повышении температуры может быть влияние поверхностного инверсионного $n$-слоя на гальваномагнитные свойства сплавов. Дело в том, что зависимости $R_{\mathrm{H}}(T)$, подобные обнаруженным нами, и даже с двойной инверсией знака коэффициента Холла (с $n-p$ - и затем $p-n$-инверсией), наблюдались ранее и в других узкощелевых полупроводниках - в $p$-InAs [34-37], $p$-InSb [38-40], $p$ - $\mathrm{Hg}_{1-x} \mathrm{Cd}_{x} \mathrm{Te}$ $(x=0.2)$ [41]. Эти эффекты объяснялись существованием на всех гранях образцов инверсионного поверхностного слоя $n$-типа проводимости, связанного, скорее всего, с адсорбцией примеси на поверхности, отделенного $p-n$-переходом от объема образца и шунтирующего объем при низких температурах. Изменение параметров этого слоя или объема образцов при внешних воздействиях (при дополнительной обработке поверхности, облучении быстрыми электронами и протонами, изменении степени легирования, а также под давлением) позволяло существенно сдвигать точку $n-p$-инверсии в сторону высоких температур, а также сближать точки $n-p$ - и $p-n$-инверсии на зависимостях $R_{\mathrm{H}}(T)$ вплоть до их перекрытия и появления на их месте лишь небольшого локального минимума.

Можно предположить, что и в нашем случае электронный тип проводимости при низких температурах и $n-p$-инверсия типа проводимости с ростом температуры также связаны с возникновением поверхностного инверсионного $n$-слоя при легировании теллурида свинца никелем. Качественное подобие этих ситуаций усиливается тем фактом, что так же, как и в нашем случае, в исследованных ранее полупроводниках наличие поверхностного слоя сильно искажало температурные зависимости коэффициента Холла (одна или две инверсии знака $R_{\mathrm{H}}$ ), но никак не отражалось на температурных зависимостях сопротивления, на которых отчетливо наблюдались участки прыжковой проводимости, примесной и собственной ионизации. Кроме того, в этом случае, по крайней мере качественно, становятся понятными пониженные значения подвижности электронов и аномально высокие значения поперечного магнитосопротивления при низких температурах. Эти аномалии, как и $n$-p-инверсия, могут быть связаны с наличием нескольких конкурирующих механизмов проводимости в исследованных образцах $\mathrm{Pb}_{1-y} \mathrm{Ni}_{y} \mathrm{Te}$ (проводимости $n$-типа в поверхностном слое, зонной проводимости $p$-типа и собственной проводимости в объеме).

\section{2. Модель электронной структуры $\mathrm{Pb}_{1-y} \mathrm{Ni}_{y} \mathrm{Te}$}

Учитывая перечисленные экспериментальные факты и рассмотренный выше вариант объяснения аномальных температурных зависимостей коэффициента Холла в сплавах, мы считаем, что непротиворечивое объяснение 


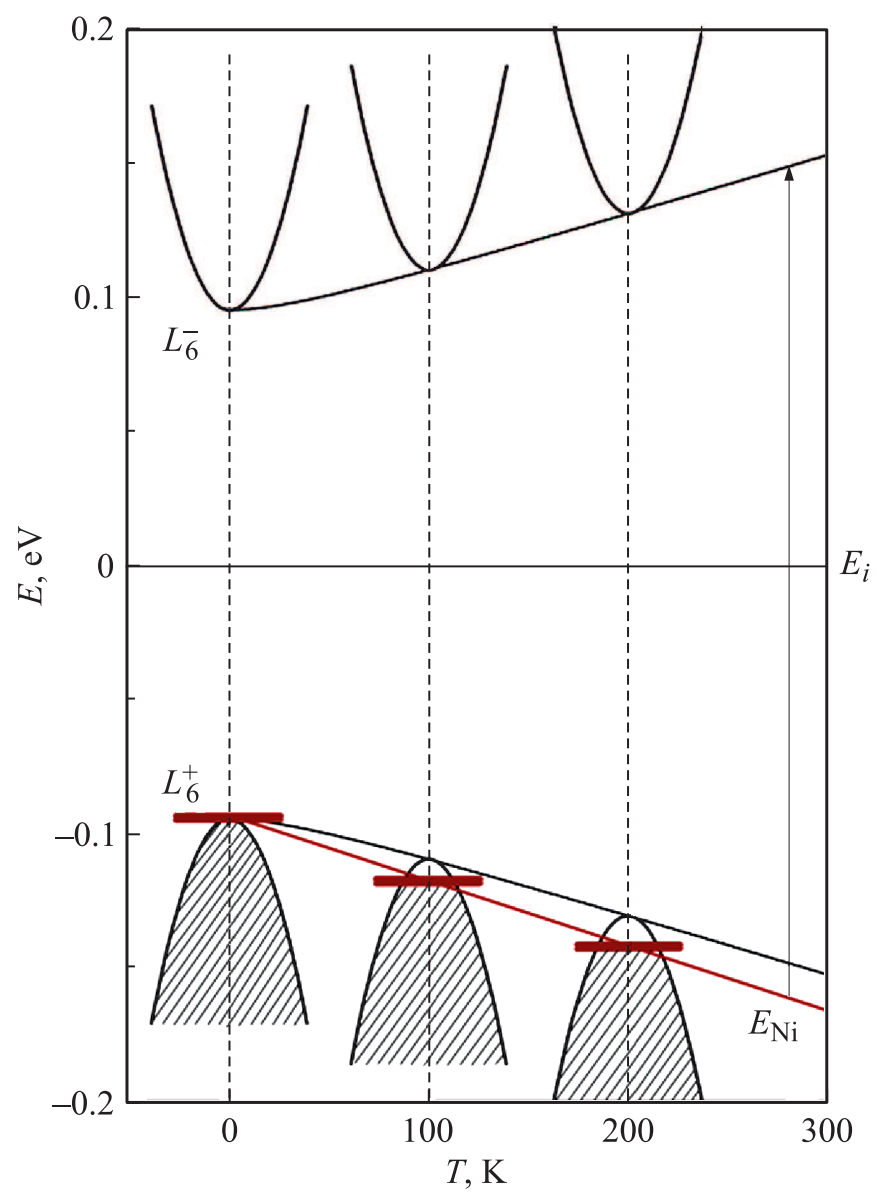

Рис. 6. Модель перестройки электронной структуры $\mathrm{Pb}_{1-y} \mathrm{Ni}_{y} \mathrm{Te}$ при увеличении температуры.

основных полученных результатов возможно в рамках следующей модели электронной структуры $\mathrm{Pb}_{1-y} \mathrm{Ni}_{y} \mathrm{Te}$ (рис. 6).

Мы предполагаем, что глубокий донорный уровень $\mathrm{Ni}$ при гелиевых температурах стабилизирует уровень Ферми на самом краю валентной зоны. При этом основными механизмами проводимости оказываются активационная проводимость по примесной зоне никеля и поверхностная проводимость $n$-типа, определяющая отрицательный знак коэффициента Холла. При увеличении степени легирования и повышении температуры примесная зона $\mathrm{Ni}$ медленно смещается вниз относительно потолка валентной зоны. Поэтому в результате перетекания электронов из валентной зоны на незаполненные примесные состояния происходит постепенное увеличение концентрации дырок в валентной зоне, приводящее к уменьшению удельного сопротивления при низких температурах и $n-p$-инверсии знака коэффициента Холла при температурах существенно ниже значений, характерных для собственной ионизации в РbTe соответственно. Дальнейшее повышение температуры должно вызывать собственную генерацию носителей заряда и $p-n$-инверсию, типичную для нелегированных сплавов на основе $\mathrm{PbTe}$.
В рамках этой модели аномалии магнитосопротивления можно, по крайней мере качественно, связать с поверхностной проводимостью $n$-типа и наличием нескольких конкурирующих механизмов проводимости в образцах. Кроме того, переход к дырочной проводимости при температурах ниже характерных для собственной проводимости, выглядит более обоснованным, поскольку уровень Ферми во всем температурном интервале находится в валентной зоне. Наконец, предположение о расположении уровня никеля вблизи потолка валентной зоны, т.е. примерно там же, где находится резонансный уровень $\mathrm{Fe}$ в $\mathrm{PbTe}$, вполне соответствует общим представлениям об электронной структуре РbTe с примесями переходных $3 d$-металлов, представленным выше.

Отметим, что пока трудно дать однозначную интерпретацию всех полученных результатов, в частности аномального поведения магнитосопротивления, а дополнительная информация об энергетическом положении примесной зоны никеля в РbTe может быть получена при исследовании легированных никелем сплавов на основе $\mathrm{PbTe}$ в условиях изменения ее положения относительно краев энергетических зон при вариации состава матрицы или под давлением.

\section{5. Заключение}

Исследованы фазовый и элементный составы образцов из монокристаллического слитка $\mathrm{Pb}_{1-y} \mathrm{Ni}_{y} \mathrm{Te}$, синтезированного методом Бриджмена-Стокбаргера. В ряде образцов обнаружены микроскопические включения второй фазы, близкой по составу к NiTe. Показано, что растворимость примеси никеля не превышает 0.35 мол\%, что не позволяет надежно определить характер распределения примеси вдоль слитка.

На температурных зависимостях гальваномагнитных параметров $\mathrm{Pb}_{1-y} \mathrm{Ni}_{y} \mathrm{Te}$ обнаружен ряд характерных аномалий, не наблюдавшихся ранее в нелегированных и легированных примесями переходных $3 d$-металлов сплавах на основе PbTe: отрицательный знак при низких температурах и $n$-p-инверсия знака коэффициента Холла $R_{\mathrm{H}}$ при температурах $170-220 \mathrm{~K}$; аномально высокие значения $\left|R_{\mathrm{H}}\right|\left(7 \cdot 10^{2}-10^{4} \mathrm{~cm}^{3} /\right.$ Кл) и относительного поперечного магнитосопротивления $\left(\Delta \rho / \rho_{0} \approx 170\right.$ в поле $B \approx 7$ Тл) при низких температурах; линейная полевая зависимость $\Delta \rho / \rho_{0}(B)$ при $T=4.2 \mathrm{~K}$ вплоть до $B \approx 7$ Тл. По температурным зависимостям сопротивления $\rho(100 / T)$ определены величины энергий активации $\Delta E_{1} \approx 0.2$ мэВ и $\Delta E_{2} \approx 85$ мэВ, которые могут соответствовать прыжковой проводимости по примесной зоне никеля и примесной или собственной ионизации.

На основании подобия результатов наших исследований и известных литературных данных для узкощелевых полупроводников $p$-InAs, $p$-InSb и $p$ - $\mathrm{Hg}_{1-x} \mathrm{Cd}_{x} \mathrm{Te}$ сделано предположение о возникновении поверхностного инверсионного $n$-слоя при легировании теллурида свинца никелем. Показано, что обнаруженные в работе 
аномалии могут быть связаны с наличием нескольких конкурирующих механизмов проводимости: электронной проводимости в поверхностном слое, дырочной и собственной проводимости в объеме образцов. Предложена модель электронной структуры $\mathrm{Pb}_{1-y} \mathrm{Ni}_{y} \mathrm{Te}$, предполагающая пиннинг уровня Ферми в пределах примесной зоны $\mathrm{Ni}$, расположенной на краю валентной зоны и постепенно смещающейся в глубь нее при увеличении концентрации никеля и с ростом температуры.

\section{Финансирование работы}

Работа выполнена при финансовой поддержке Российского фонда фундаментальных исследований (проекты № 16-02-00865 и № 19-02-00774).

\section{Конфликт интересов}

Авторы заявляют, что у них нет конфликта интересов.

\section{Список литературы}

[1] E.P. Skipetrov, A.V. Knotko, E.I. Slynko, V.E. Slynko. Low Temp. Phys., 41, 141 (2015).

[2] E.P. Skipetrov, L.A. Skipetrova, A.V. Knotko, E.I. Slynko, V.E. Slynko. J. Appl. Phys., 115, 133702 (2014).

[3] M.N. Vinogradova, E.A. Gurieva, V.I. Zharskii, S.V. Zarubo, L.V. Prokofeva, T.T. Dedegkaev, I.I. Kryukov. Sov. Phys. Semicond., 12, 387 (1978).

[4] A.A. Vinokurov, S.G. Dorofeev, O.I. Tananaeva, A.I. Artamkin, T.A. Kuznetsova, V.P. Zlomanov. Inorg. Mater., 42, 1318 (2006).

[5] V.D. Vulchev, L.D. Borisova, K. Dimitrova. Phys. Status Solidi A, 97, K79 (1986).

[6] E.P. Skipetrov, O.V. Kruleveckaya, L.A. Skipetrova, E.I. Slynko, V.E. Slynko. Appl. Phys. Lett., 105, 022101 (2014).

[7] E.P. Skipetrov, B.B. Kovalev, L.A. Skipetrova, A.V. Knotko, V.E. Slynko. J. Alloys Compd., 775, 769 (2019).

[8] E.P. Skipetrov, B.B. Kovalev, L.A. Skipetrova, A.V. Knotko, V.E. Slynko. Low Temp. Phys., 45, 233 (2019).

[9] A.D. LaLonde, Y. Pei, H. Wang, G.J. Snyder. Mater. Today, 14, 526 (2011).

[10] J. Androulakis, I. Todorov, D.-Y. Chung, S. Ballikaya, G. Wang, C. Uher, M. Kanatzidis. Phys. Rev. B, 82, 115209 (2010).

[11] Y. Pei, X. Shi, A. LaLonde, H. Wang, L. Chen, G.J. Snyder. Nature, 473, 66 (2011).

[12] Y. Pei, H. Wang, G.J. Snyder. Adv. Mater., 24, 6125 (2012).

[13] L.-D. Zhao, V.P. Dravid, M.G. Kanatzidis. Energy Environ. Sci., 7, 251 (2014).

[14] X. Zhang, L.-D. Zhao. J. Materiomics, 1, 92 (2015).

[15] A.M. Dehkordi, M. Zebarjadi, J. He, T.M. Tritt. Mater. Sci. Eng. R, 97, 1 (2015).

[16] G.D. Mahan, J.O. Sofo. Proc. Natl. Acad. Sci. USA, 93, 7436 (1996).

[17] J.P. Heremans, V. Jovovic, E.S. Toberer, A. Saramat, K. Kurosaki, A. Charoenphakdee, S. Yamanaka, G.J. Snyder. Science, 321, 554 (2008).

[18] J.P. Heremans, B. Wiendlocha, A.M. Chamoire. Energy Environ. Sci., 5, 5510 (2012).

[19] B. Wiendlocha. Phys. Rev. B, 97, 205203 (2018).
[20] K.A. Kikoin, V.N. Fleurov. Transition Metal Impurities in Semiconductors: Electronic Structure and Physical Properties (Singapore, World Scientific, 1994).

[21] G.V. Lashkarev, M.V. Radchenko, V.V. Asotskiy, A.V. Brodovoy, A.I. Mirets, O.I. Tananaeva. Mater. Sci. Forum, 182-184, 631 (1995)

[22] V.V. Asotskii, T.A. Kuznetsova, G.V. Lashkarev, M.V. Radchenko, O.I. Tananaeva, V.V. Teterkin. Semiconductors, 30, 88 (1996).

[23] T.A. Kuznetsova, V.P. Zlomanov, O.I. Tananaeva. Inorg. Mater., 34, 878 (1998).

[24] D. Lucovic, P.M. Nikolic, S. Vujatovic, S. Savic, D. Urosevic. Sci. Sintering, 39, 161 (2007).

[25] N. Romcevic, J. Trajic, T.A. Kuznetsova, M. Romcevic, B. Hadzic, D.R. Khokhlov. J. Alloys Compd., 442, 324 (2007).

[26] N. Romcevic, J. Trajic, M. Romcevic, D. Stojanovic, T.A. Kuznetsova, D.R. Khokhlov, W. Dobrowolski, R. Rudolf, I. Anzel. Acta Phys. Pol. A, 115, 805 (2009).

[27] N. Romcevic, J. Trajic, M. Romcevic, D. Stojanovic, T.A. Kuznetsova, D.R. Khokhlov, W.D. Dobrowolski. Optoelectronics Adv. Mater-Rapid Commun., 4, 470 (2010).

[28] E.P. Skipetrov, O.V. Kruleveckaya, L.A. Skipetrova, A.V. Knotko, E.I. Slynko, V.E. Slynko. J. Appl. Phys., 118, 195701 (2015).

[29] V.E. Slyn'ko. Visn. Lviv Univ., Ser. Physic., No. 34, 291 (2001).

[30] V.E. Slynko, W. Dobrowolski. Visn. Lviv Polytec. Natl. Univ., Electronics, No. 681, 144 (2010).

[31] E.P. Skipetrov, N.A. Pichugin, E.I. Slyn'ko, V.E. Slyn'ko. Low Temp. Phys., 37, 210 (2011).

[32] E.P. Skipetrov, A.N. Golovanov, A.V. Knotko, E.I. Slyn'ko, V.E. Slyn'ko. Semiconductors, 46, 741 (2012).

[33] G. Nimtz, B. Schlicht. In: Narrow-Gap Semiconductors [Springer Tracts in Modern Physics (Springer, BerlinHeidelberg-N.Y.-Tokyo, 1983) v. 98].

[34] V.V. Voronkov, E.V. Soloveva, M.I. Iglitsyn, M.N. Pivovarov. Sov. Phys. Semicond., 2, 1499 (1969).

[35] O.K. Gusev, V.P. Kireenko, A.A. Lomtev, V.B. Yarzhembitskii. Sov. Phys. Semicond., 17, 727 (1983).

[36] G.Y. Andersen, O.K. Gusev, F.A. Zaitov, V.P. Kireenko, V.B. Yarzhembitskii. Sov. Phys. Semicond., 25, 1205 (1991).

[37] G.I. Koltsov, Yu.V. Krutenyuk, E.P. Skipetrov. In: Ion Implantation Technology - 94, ed. by S. Coffa, G. Ferla, F. Priolo, E. Rimini (Amsterdam, Elsevier Science B.V., 1995) p. 835 .

[38] E.W. Kreutz. Phys. Status Solidi A, 77, 583 (1983).

[39] N.B. Brandt, V.V. Dmitriev, E.A. Ladygin, E.P. Skipetrov. Sov. Phys. Semicond., 21, 315 (1987).

[40] E.P. Skipetrov, V.V. Dmitriev, G.I. Koltsov, E.A. Ladygin,. Fiz. Tekh. Poluprovodn., 21, 962 (1987).

[41] W. Scott, R.J. Hager. J. Appl. Phys., 42, 803 (1971).

Редактор Л.В. Шаронова 


\title{
Effect of nickel impurity on \\ galvanomagnetic properties and \\ electronic structure of $\mathrm{PbTe}$
}

\author{
E.P. Skipetrov ${ }^{1,2}$, B.B. Kovalev ${ }^{1}$, I.V. Shevchenko ${ }^{1}$, \\ A.V. Knotko ${ }^{2,3}$, V.E. Slynko ${ }^{4}$ \\ ${ }^{1}$ Lomonosov Moscow State University \\ (Faculty of Physics), \\ 119991 Moscow, Russia \\ ${ }^{2}$ Lomonosov Moscow State University \\ (Faculty of Materials Science), \\ 119991 Moscow, Russia \\ ${ }^{3}$ Lomonosov Moscow State University \\ (Faculty of Chemistry), \\ 119991 Moscow, Russia \\ ${ }^{4}$ Frantsevich Institute for Problems \\ of Materials Science, \\ National Academy of Sciences of Ukraine, \\ 58001 Chernivtsi, Ukraine
}

Abstract The phase and elemental composition and galvanomagnetic properties (at temperatures $4.2 \leq T \leq 300 \mathrm{~K}$, magnetic fields $B \leq 7 \mathrm{~T}$ ) of $\mathrm{Pb}_{1-y} \mathrm{Ni}_{y} \mathrm{Te}$ alloys are studied at variation of the nickel impurity concentration along the single-crystal ingot synthesized by the Bridgman-Stockbarger technique. It is shown that the solubility of nickel does not exceed $0.35 \mathrm{~mol} \%$. Anomalous temperature dependences of the Hall coefficient and temperature and field dependences of magnetoresistance are found. To explain the results, it is assumed that an n-type inversion layer appears on the surface of the samples and that there are several competing conduction mechanisms in the samples. The model of the $\mathrm{Pb}_{1-y} \mathrm{Ni}_{y} \mathrm{Te}$ electronic structure is proposed that assumes pinning of the Fermi level within the Ni impurity band, located at the edge of the valence band and moving deeper into it with increasing temperature. 